\title{
EOS of Neutron Matter and Neutron Star Properties
}

\author{
Kh. S.A. Hassaneen ${ }^{1 \& 2}$, H.M. Abou-Elsebaa ${ }^{1}$, E.A. Sultan ${ }^{1}$ and N.N. Abd Allah ${ }^{1}$ \\ ${ }^{1}$ Physics department, Faculty of Science, Sohag University, Sohag, Egypt. \\ ${ }^{2}$ Physics department, Faculty of Science, Taif University, Taif, Saudi Arabia.
}

Rec. 18 Jan, 2015 Accept. 5 Feb, 2015

\begin{abstract}
The Equation of State (EOS) of pure neutron matter at zero temperature is calculated up to five saturation densities within the Brueckner theory with the inclusion of three-body forces. Three different realistic and accurate two-body forces are considered to evaluate the G-matrix effective interaction for nuclear matter. These models are the chiral $\mathrm{N}^{3} \mathrm{LO}$, the CD-Bonn and the Argonne $\mathrm{V}_{18}$, which give quite different EOS. Two types of three-body forces are included to the effective interaction, which might be important at densities several times that of nuclear matter density. Using a microscopic EOS for pure neutron matter, static properties of non-rotating neutron stars such as masses and radii are evaluated. The resulting maximum masses of neutron stars using different interactions near $2 \mathrm{M}_{\odot}$ are found to be in reasonable agreement with the measured ones PSR J1614-2230 (with $\mathrm{M}_{\max }=1.97 \pm 0.04 \mathrm{M}_{\odot}$ ) and PSR J0348+0432 (with $\mathrm{M}_{\max }=2.01 \pm 0.04$ $\mathrm{M}_{\odot}$ ).
\end{abstract}

Keywords: Pure neutron matter, three-body force, contact term, neutron star properties.

\section{Introduction:}

The equation of state (EOS) for neutron star matter and infinite nuclear matter has been intensively studied for many years (see, for example, Refs. (Pethick, et al., 1995; Hansen, et al., 1995). A correct description of the EOS would have far reaching consequences for topics ranging from the cooling of neutron stars (Pethick, et al., 1995; Prakash, 1994) to the heavy ion collision physics (B.-A. Li, C.M. Ko and Ren, Z. 1997). Furthermore, experiments of radioactive ion beam (Tanihata, 1995; Hansen, et al., 1995). have provided new information on the structure of unstable nuclei far from equilibrium. The latter may open the possibility of extracting information on the EOS for asymmetric matter and the density dependence of the nuclear symmetry energy. Moreover, neutron stars are macroscopic objects where the stability is guaranteed by the Pauli principle of nucleons (together with a repulsive short-range interaction). Therefore, the structure of a neutron star is dictated by the strong interaction (and, of course, gravity). The key ingredient that enters the stability condition is precisely the equation of state (Leupold, et al., 2011).

Recently, high-quality observational data of neutron stars set new stringent constraints for the EOS of cold and dense matter, otherwise inaccessible by experiment. The masses of two heavy pulsars have been determined with high precision. One of those is the radio pulsar (PSR) J1614-2230 with a mass $\mathrm{M}=(1.97 \pm$ 0.04) $\mathrm{M}_{\odot}$ (Demorest, et al., 2010). and the other is the radio pulsar PSR J0348+0432 accurately determined mass with $\mathrm{M}=(2.01 \pm$ 0.04) $\mathrm{M}_{\odot}$ (Antoniadis et al., 2013). where $\mathrm{M}_{\odot}$ denotes the mass of the sun. Only a sufficiently stiff EOS can support such neutron stars against gravitational collapse. Whereas neutron star radii are much less accurately known, the combination of available data makes these objects nonetheless an indispensable tool to constrain possible EOS (Hell, et al., 2014).

(Tews et al., 2013). have done perturbation-theory calculations for pure neutron matter (PNM) with nuclear interactions derived from chiral perturbation theory including the full next-to-next-to-nextto-leading order $\left(\mathrm{N}^{3} \mathrm{LO}\right)$ contribution, with

\footnotetext{
* Corresponding author:

Dr. N.N. Abd Allah

$\bowtie$ nabil.abdallah@science.sohag.edu.eg
} 67 
three- and four-body forces. Compared to calculations with three-body interactions defined only to next-to-next-to-leading order (NNLO), the inclusion of all $\mathrm{N}^{3} \mathrm{LO}$ diagrams was found to be very important for nuclear structure and reactions. Their results provide constraints for the nuclear equation of state and for neutron-rich matter in astrophysics. In the present work, the $\mathrm{N}^{3} \mathrm{LO}$ and NNLO potentials complemented by phenomenological Urbana Three body forces (TBFs) (Baldo, et al., 2008). instead of chiral three-nucleon forces, have been applied in calculations to neutron matter.

In this work we will derive the EOS of PNM by many-body theory, derived from different realistic nucleon-nucleon $(\mathrm{NN})$ interactions such as the CD-Bonn potential (Machleidt, 2001). the $\mathrm{N}^{3} \mathrm{LO}$ potential (Entem, et al., 2003). and the Argonne $\mathrm{V}_{18}$ potential (Wiringa, et al., 1995). The many-body approaches are used, as the BruecknerHartree-Fock (BHF) approach with the inclusion of three-body force (Baldo, et al., 2008). or contact-term interaction to give more repulsive EOS (Khaled et al., 2013).

The plan of the paper is the following. The main features of BHF approach used in this work including two types of three-body forces are shown in the following section. The results for the neutron matter equation of state are presented and discussed in Sec. 3. In Sec. 4 neutron star matter is examined and discussed in view of the new observational constraints. Finally, the conclusions and perspectives for the present work are outlined in Sec. 5.

\section{BHF approach:}

One main feature of the BHF approach to nuclear matter is that the binding energy and related quantities such as the self-energy, i.e. mass operator, which can be developed according to the so-called hole-line expansion. Its expression is

$$
\boldsymbol{\Sigma}(\mathbf{k}, \mathbf{e})=\sum_{\mathbf{k}^{\prime}<\mathbf{k}_{\mathbf{F}}}\left\langle\mathbf{k} \mathbf{k}^{\prime}\left|\mathbf{G}\left(\mathbf{e}(\mathbf{k})+\mathbf{e}\left(\mathbf{k}^{\prime}\right)\right)\right| \mathbf{k k}^{\prime}\right\rangle_{\mathbf{A}}
$$

where the subscript A means that the G-matrix has to be anti-symmetrized.

The auxiliary single-particle energy e $(\mathrm{k})$ is defined below according to the scheme of the iterative solution of the Bethe-Goldstone equation. If one chooses only $\mathrm{U}(\mathrm{k})=\mathrm{Re} \Sigma(\mathrm{k}, \mathrm{e})$ as auxiliary potential, then single-particle energy has the form:

$e(k)=\frac{\hbar^{2} k^{2}}{2 m}+U(k)$

First we solve the Bethe-Goldstone equation

$G(\omega, k)=V+\sum_{k_{a} k_{b}} V \frac{\left|k_{a} k_{b}\right\rangle Q\left\langle k_{a} k_{b}\right|}{\omega-e\left(k_{a}\right)-e\left(k_{b}\right)+i \eta} G(\omega, k)$

where $\mathrm{V}$ is the bare nucleon-nucleon interaction, $\omega=\mathrm{e}(\mathrm{k})+\mathrm{e}\left(\mathrm{k}^{\prime}\right)$ is the starting energy, and $\mathrm{Q}$ is the Pauli operator, which prevents two particles to scatter inside the Fermi sphere. Using the continuous choice (Jeukenne et al., 1976). the auxiliary potential $\mathrm{U}(\mathrm{k})$ has been self-consistently evaluated along with the G-matrix from Eq. 3. Furthermore, within BHF approach, one can easily evaluate the binding energy per nucleon for nuclear matter:

$\frac{E(k)}{A}=\frac{3}{5} \frac{k_{F}^{2}}{2 m}+\frac{1}{2 \rho} \operatorname{Re} \sum_{k^{\prime} \leq k_{F}}\left\langle k k^{\prime}\left|G\left(e(k)+e\left(k^{\prime}\right)\right)\right| k k^{\prime}\right\rangle_{a}$

where $\mathrm{k}_{\mathrm{F}}$ is the Fermi momentum.

We use two methods to make the EOS for pure neutron matter be more repulsive. First method is done by adding the three-bodyforce. The phenomenological TBF model consists of an attractive term $V_{i j k}^{2 \pi}$ due to twopion exchange with the excitation of an intermediate $\Delta$-resonance, and a repulsive phenomenological term $V_{i j k}^{R}$

$V_{i j k}=V_{i j k}^{2 \pi}+V_{i j k}^{R}$

Where $V_{i j k}$ is the three body interaction, the first term is the so-called two-pion exchange contribution. It is a cyclic sum over the nucleon indices $i, j, k$ of products of anticommutator $\{$,$\} and commutator [$,$] terms$

$V_{i j k}^{2 \pi}=A \sum_{c y c}\left(\left\{X_{i j}, X_{j k}\right\}\left\{\tau_{i} \cdot \tau_{j}, \tau_{j} \cdot \tau_{k}\right\}+\frac{1}{4}\left[X_{i j}, X_{j k}\right]\left[\tau_{i} \cdot \tau_{j}, \tau_{j} \cdot \tau_{k}\right]\right)$

Where:

$X_{i j}=Y\left(r_{i j}\right) \sigma_{i} \cdot \sigma_{j}+T\left(r_{i j}\right) \cdot S_{i j}$

is the one-pion exchange operator, $\sigma$ and $\tau$ are known as the Pauli spin and isospin operators, and 
$S_{i j}=3\left[\left(\sigma_{i} \cdot r_{i j}\right)\left(\sigma_{j} \cdot r_{i j}\right)-\sigma_{i} \cdot \sigma_{j}\right]$

is called the tensor operator. Both $\mathrm{Y}(\mathrm{r})$ and $\mathrm{T}(\mathrm{r})$ are the Yukawa and tensor functions associated to the one-pion exchange as in the two-body potential. The repulsive term is written as.

$V_{i j k}^{R}=U \sum_{c y c} T^{2}\left(r_{i j}\right) \cdot T^{2}\left(r_{j k}\right)$

(8)

The strengths $A$ and $U$ are parameters that in the present work are adjusted to reproduce the exact saturation point of symmetric nuclear matter.

We introduced the Urbana three-nucleon model within the BHF approach. To incorporate the TBF in the Brueckner scheme we followed the method of (Lejeune et al., 1986). The TBF is reduced to an effective twobody force by averaging on the position of the third particle, assuming that the probability of having two particles at a given distance is reduced according to the two-body correlation function. The resulting effective two-body force is of course density dependent. We have adjusted the parameters $\mathrm{A}$ and $\mathrm{U}$ in order to reproduce the correct saturation point of symmetric nuclear matter and the EOS become much more repulsive at high density, since the high density region is needed in neutron star studies.

The second method to achieve saturation in nuclear matter is the effective interaction or the self-energy of BHF calculations by a simple contact interaction, which we have chosen following to the notation of the Skyrme interaction to be in the form (Gögelein et al., 2009).

$\frac{\Delta H}{\rho}=C T=\frac{1}{2} t_{0} \rho+\frac{1}{12} t_{3} \rho^{1+\alpha}$

(9)

where $\rho$ is the density and $t_{0}, t_{3}$ and $\alpha$ are free parameters. For symmetric nuclear matter with $\alpha=0.5$, we have fitted $t_{0}$ and $t_{3}$ in such a way that BHF calculations plus the contact term (CT) yield the empirical saturation point for symmetric nuclear matter. In Table (1) we show the values of $t_{0}$ and $t_{3}$ for CD-Bonn potential, $\mathrm{N}^{3} \mathrm{LO}$ potential and Argonne $\mathrm{V}_{18}$ potential.

\section{EOS for pure neutron matter:}

The energy per nucleon of neutron matter is the only input required for determining the mass-radius relationship for neutron stars, and hence the ranges of physically allowable neutron star masses. In Figure 1. we show the results for the energy per nucleon in the case of a system composed of neutrons only. For three choices of the $N N$ potential, curves of EOS with two types TBF are displayed supplemented to BHF approach. When phenomenological TBF are used, the parameters $A$ and $U$ are fixed by the calculation of saturation properties in the symmetric case.

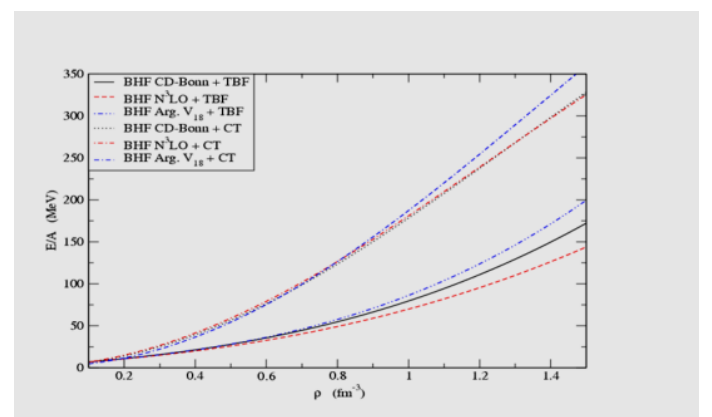

Figure 1. The equation of state $\mathrm{E} / \mathrm{A}$ in $\mathrm{MeV}$ for pure neutron matter as a function of density $\rho$ in $\mathrm{fm}^{-3}$ for different potentials. The potentials employed are the CD-Bonn + TBF (solid line), the CD-Bonn + CT (dotted line), $\mathrm{N}^{3} \mathrm{LO}+\mathrm{TBF}$ (short dashed line), $\mathrm{N}^{3} \mathrm{LO}+\mathrm{CT}$ (dot-dashed line), the Argonne $\mathrm{V}_{18}$ potential $+\mathrm{TBF}$ (double dot-dashed line) and Argonne $\mathrm{V}_{18}$ potential + CT (double dashed dot line).

\begin{tabular}{|c|c|c|c|}
\hline Parameters & CD-Bonn & $\mathbf{N}^{3} \mathbf{L O}$ & Arg. $\mathbf{V}_{\mathbf{1 8}}$ \\
\hline $\mathrm{t}_{0}$ & -136.2609 & -131.8981 & -181.4014 \\
\hline $\mathrm{t}_{3}$ & 2440.734 & 2538.8274 & 2689.352 \\
\hline
\end{tabular}

Table I: The values of the parameters $\mathrm{t}_{0}$ and $\mathrm{t}_{3}$ that are used to fit the saturation point.

The introduction of TBF does not change qualitatively the density dependence of $E / A$ but makes the EOS more stiff. The same behavior is noticed when contact interaction is added to the effective interaction to yield the empirical saturation point for symmetric nuclear matter with the same parameters defined in Table I.

We find agreement at low densities between all interactions extend to very high densities especially for CD-Bonn + CT and $\mathrm{N}^{3} \mathrm{LO}+\mathrm{CT}$ presented by dotted and dot-dashed 
lines and they are non-local potentials.

In contrast to EOS results with supplemented phenomenological terms, there are agreements at low densities between all interactions because the strong tensor effect in the ${ }^{3} S_{1}-{ }^{3} D_{1}$ channel is absent. While, there are noticeable differences at very high densities for all interactions. The EOS for the Argonne $\mathrm{V}_{18}+\mathrm{CT}$ appears the most repulsive than the other potentials. Chiral $\mathrm{N}^{3} \mathrm{LO}+\mathrm{TBF}$ EOS that seem to provide a very soft pure neutron matter EOS. Moreover, there is another indication one can see it from Figure 1. We observe that the EOS for pure neutron matter takes only positive values which means that it is unbound. The energy per nucleon is rising approximately monotonically with increasing density, which is in agreement with the manybody calculations done by (Kohno, 2013).

\section{Neutron star structure:}

The structure of a neutron star is characterized by its mass and radius. Additional parameters of interest are the moment of inertia and the crust thickness; these are important for the dynamics and transport properties of pulsars. In the present work, we assume that a neutron star is a spherically symmetric distribution of mass in hydrostatic equilibrium. The effects of rotations can be neglected; assuming there is no magnetic field. Then the equilibrium configurations are simply obtained by solving the Tolman - Oppenheimer - Volkoff (TOV) equations [Oppenheimer et al., 1939; Tolman, 1934).

$$
\begin{aligned}
& \frac{d P(r)}{d r}=-\frac{G m(r) \rho(r)}{r^{2}}\left\{\frac{\left(1+\frac{P(r)}{c^{2} \rho(r)}\right)\left(1+\frac{4 \pi r^{3} P(r)}{c^{2} m(r)}\right)}{\left(1-\frac{G m(r)}{r c^{2}}\right)}\right\} \\
& \frac{d m(r)}{d r}=4 \pi r^{2} \rho(r)
\end{aligned}
$$

where $\mathrm{P}(\mathrm{r})$ and $\rho$ are the pressure and density respectively at radius $\mathrm{r}, \mathrm{m}(\mathrm{r})$ is the gravitational mass inside $r, G=1.327 \times 10^{11}$ $\mathrm{km}^{3} /\left(\mathrm{M}_{\odot} \cdot \mathrm{s}^{2}\right)$ is the universal constant of gravitation. The TOV equations are first order differential equations, which can be integrated by the following boundary conditions:

$\mathrm{m}(0)=0.0, \mathrm{P}(\mathrm{R})=\mathrm{P}_{\text {surf }}$

The first condition means that the density $\rho$ and the pressure $\mathrm{P}(\mathrm{r})$ are finite at the center of the neutron star, the second condition determine the pressure at the surface of the neutron star. Starting with a central density $\rho(r=0) \equiv \rho_{c}$ the solutions of these equations are obtained by integrating them out from the neutron-star center till its edge where $\mathrm{P}$ is zero. This gives the stellar radius $\mathrm{R}$ and the gravitational mass is then

$M_{G}=m(R)=4 \pi \int_{0}^{R} d r r^{2} \rho(r)$

It turns out that the mass of the neutron star has a maximum value as a function of radius (or central density) above which the star is unstable against collapse to a black hole. The value of the maximum mass depends on the nuclear EOS (Taranto, et al., 2013).

For the outer part of the neutron star $(\rho<$ $0.001 \mathrm{fm}^{-3}$ ) we have used the equations of state by (Baym et al., 1971). whereas for the middle-density regime $\left(0.001 \mathrm{fm}^{-3}<\rho<0.08\right.$ $\mathrm{fm}^{-3}$ ), the results of (Lorenz et al. 1993). are used. Furthermore the present equations of states for pure neutron matter with the highdensity EOSs $\left(\rho>0.08 \mathrm{fm}^{-3}\right)$ employed to describe the core of the neutron star.

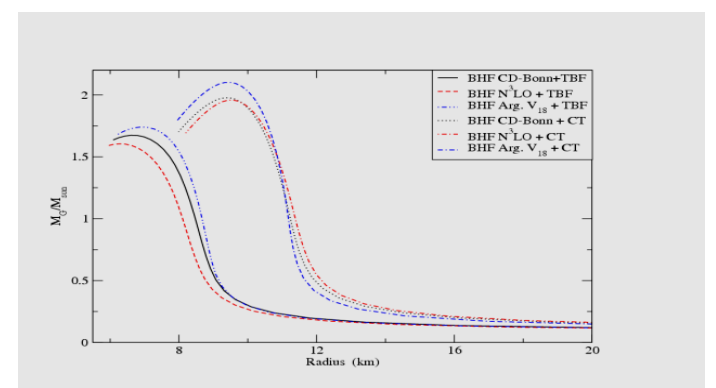

Fig 2. Predicted mass-radius relations of neutron stars as a function of the stellar radius in $\mathrm{km}$ for different potentials. The potentials employed are the CD-Bonn + TBF (solid line), the CD-Bonn + CT (dotted line), $\mathrm{N}^{3} \mathrm{LO}+\mathrm{TBF}$ (short dashed line), $\mathrm{N}^{3} \mathrm{LO}+\mathrm{CT}$ (dot-dashed line), the Argonne $\mathrm{V}_{18}$ potential + TBF (double dot-dashed line) and Argonne $\mathrm{V}_{18}$ potential $+\mathrm{CT}$ (double dashed dot line).

Figure 2. shows the calculated mass-radius relations of neutron stars. Consistent with the EOSs in Figure 1, the maximum mass of the neutron star and the corresponding radius given by $\mathrm{N}^{3} \mathrm{LO}+\mathrm{TBF}$ are the smallest, and those obtained by Argonne $\mathrm{V}_{18}+\mathrm{CT}$ are the largest. The results for CD-Bonn $+\mathrm{CT}$ are 
similar to $\mathrm{N}^{3} \mathrm{LO}+\mathrm{CT}$ but its calculations for both mass and radius are a little smaller than $\mathrm{V}_{18}+\mathrm{CT}$. In contrast, when the EOS results are supplemented by phenomenological TBF terms, there are differences observed in the maximum values of mass.

Figure 3. displays the neutron star masses as a function of the central density $\rho_{\mathrm{c}}$ of the star, with several effective interactions mentioned above. From Figure 3, it can be seen that the $\mathrm{N}^{3} \mathrm{LO}+\mathrm{TBF}$ interaction exhibits the smallest maximum mass at the largest central density $\rho_{\max }$. Table II shows the maximum mass limits $M_{\max }$, the radius $\mathrm{R}$ and the corresponding central densities $\rho_{\max }$ extracted from Figures 2 and 3.

From Table II, one finds a maximum mass of neutron star.

$$
M_{\max }=1.674 \mathrm{M}_{\odot}
$$

at a central density $\rho_{d} / \rho_{0}=25$ with a radius $R=$ $6.611 \mathrm{~km}$ for CD-Bonn + TBF model. Also we find a maximum mass of neutron star.

$$
M_{\max }=1.977 \mathrm{M}_{\odot}
$$

at a central density $\rho_{d} / \rho_{0}=11$ with a radius $R=$ $9.469 \mathrm{~km}$ for CD-Bonn + CT model. In the case of $\mathrm{N}^{3} \mathrm{LO}+\mathrm{TBF}$, one notes a maximum mass of

$$
M_{\text {max }}=1.605 \mathrm{M}_{\odot}
$$

at a central density $\rho_{d} / \rho_{0}=28$ with a radius $R$ $=6.271 \mathrm{~km}$. If we use $\mathrm{N}^{3} \mathrm{LO}+\mathrm{CT}$, we find a maximum mass of

$$
M_{\max }=1.959 \mathrm{M}_{\odot}
$$

at a central density $\rho_{\mathrm{d}} / \rho_{0}=11$ with a radius $\mathrm{R}=$ $9.559 \mathrm{~km}$. In the case of Argonne $\mathrm{V}_{18}+\mathrm{TBF}$, we find a maximum mass of neutron star.

$$
M_{\text {max }}=1.741 \mathrm{M}_{\odot}
$$

at a central density $\rho_{\mathrm{c}} / \rho_{0}=22.5$ with a radius $\mathrm{R}$ $=6.908 \mathrm{~km}$. Lastly, in the case of Argonne $\mathrm{V}_{18}$ + CT a maximum mass of neutron star has the value

$$
M_{\text {max }}=2.102 \mathrm{M}_{\odot}
$$

at a central density $\rho_{\mathrm{c}} / \rho_{0}=11$ with a radius $\mathrm{R}=$ $9.481 \mathrm{~km}$.

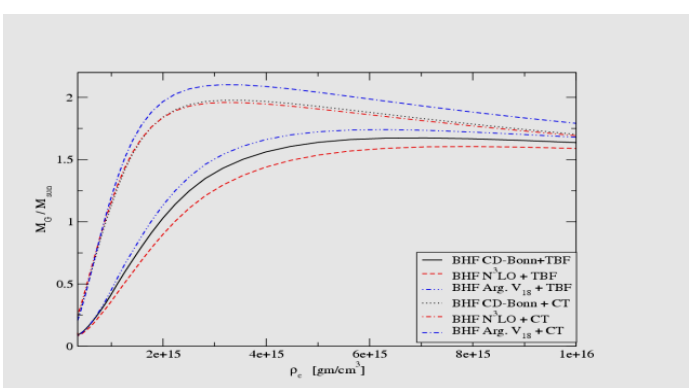

Figure 3. neutron star gravitational masses $\mathrm{M}_{\mathrm{G}}$ (in units of $\mathrm{M}_{\odot}$ ) as a function of the central density $\rho_{c}$ in units of $\mathrm{gm} / \mathrm{cm}^{3}$ for different potentials. The notation are the same as Figure 2.

Finally one can see from the figures (2 and 3 ) that neutron stars properties calculated with a stiff equation of state (e.g., CD-Bonn + CT, $\mathrm{N}^{3} \mathrm{LO}+\mathrm{CT}$ and Argonne $\mathrm{V}_{18}+\mathrm{CT}$ ) have greater maximum masses than neutron stars derived from a soft equation of state. Furthermore neutron stars derived from a stiff equation of state have a lower central density and larger radius than do neutron stars of the same mass computed from a soft equation of state.

\begin{tabular}{|l|l|l|l|}
\hline Model & $\mathrm{M}_{\max }\left(\mathrm{M}_{\odot}\right)$ & Radius $(\mathrm{km})$ & $\rho_{\mathrm{c}} / \rho_{0}$ \\
\hline CD-Bonn +TBF & 1.674 & 6.611 & 25 \\
\hline CD-Bonn + CT & 1.977 & 9.469 & 11 \\
\hline $\mathrm{N}^{3} \mathrm{LO}+\mathrm{TBF}$ & 1.605 & 6.271 & 28 \\
\hline $\mathrm{N}^{3} \mathrm{LO}+\mathrm{CT}$ & 1.959 & 9.559 & 11 \\
\hline Arg. $\mathrm{V}_{18}+\mathrm{TBF}$ & 1.741 & 6.908 & 22.5 \\
\hline Arg. $\mathrm{V}_{18}+\mathrm{CT}$ & 2.102 & 9.481 & 11 \\
\hline
\end{tabular}

Table II: The maximum mass, radius and $\rho_{\mathrm{c}} / \rho_{0}$ of neutron star for different models.

\section{Conclusion:}

We studied neutron star properties, in particular NS's maximum masses and radius using the BHF approximation with exact Pauli's operator with the inclusion of two types of three body forces. The three body forces used are the phenomenological TBF and a simple contact term added to the effective interaction. It is found that neutron star properties are sensitive to microscopic model calculations, this means that the maximum masses and radii depend on the stiffness of the suggested EOS. Neutron star properties calculated with a stiff EOS have a lower 
central density, a larger radius than stars of the same mass computed from a soft EOS.

\section{References:}

Pethick, C.J. and Ravenhall, D.G. (1995). Annu. Rev. Nucl. Part. Phys. 45, 429.

Prakash, M. (1994). Phys. Rep. 242, 191.

B.-A. Li, C.M. Ko and Ren, Z. (1997). Phys. Rev. Lett. 78, 1644,

Tanihata, I. (1995). Prog. Part. Nucl. Phys. 35, 505.

Hansen, P.G., Jensen A.S. and Jonson, B. Annu. Rev. Nucl. Part. Sci. 45, 591, (1995).

Leupold, S., Redlich, K., Stephanov, M., Andronic, A., Blaschke, D. et al., Lect. Notes Phys. 814, 39-334 (2011). DOI 10.1007/978-3-642-13293-3_3

Demorest, P.B., Pennucci, T., Ransom, S.M., Roberts, M.S.E. and Hessels, J.W.T., Nature 467, 1081 (2010).

Antoniadis, J., Freire, P.C., Wex, N., Tauris, T.M., Lynch, R.S., van Kerkwijk, M.H., Kramer, M., Bassa, C., Dhillon, T. Driebe V.S. et al., (2013). Science 340, 6131.

Hell, T. and Weise, W. (2014). Phys. Rev. C 90, 045801 .

Tews, I., Krüger, T., Hebeler, K. and Schwenck, A. (2013). Phys. Rev. Lett., $110,032504$.
Baldo, M. and Alaa Eldeen Shaban, (2008). Phys. Lett. B 661, 373.

Machleidt, R. (2001). Phys. Rev. C 63: 024001 .

Entem, D.R. and Machleidt, R. (2003). Phys. Rev. C 68, 041001.

Wiringa, R.B., Stoks, V.G.J. and Schiavilla, R. (1995). Phys. Rev. C 51, 38.

Khaled Hassaneen and Hesham Mansour, J. (2013). Nuc. Part. Phys. 3(4): 77-96, DOI: 10.5923/j.jnpp.20130304.05.

Jeukenne, J. P., Lejeune, A. and Mahaux, C. (1976). Phys. Rep. 25, 83.

Lejeune, A., Grange', P., Martzolff M. and Cugnon, J. (1986). Nucl. Phys. A 453, 189.

Gögelein, P., van Dalen, E.N.E., Gad, Kh., Hassaneen, Kh.S.A. and Müther, H. (2009). Phys. Rev. C79, 024308.

Kohno, M. (2013). Phys. Rev. C88, 064005.

Oppenheimer J.R. and Volkoff, G.M. (1939). Phys. Rev. 55, 374

Tolman, R.C. (1934). Proc. Nat. Acad. Sci. USA 20, 3 .

Taranto, G., Baldo M. and Burgio, G.F. (2013). Phys. Rev. C 87, 045803.

Baym, G., Pethick, C. and Sutherland, D. (1971). ApJ 170, 299.

Lorenz, C.P., Ravenhall D.G. and Pethick, C.J. (1993). Phys. Rev. Lett. 70, 379.

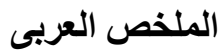

\section{معادلة الحالة للوسط النيوترونى وخواص النجوم النيوترونية}

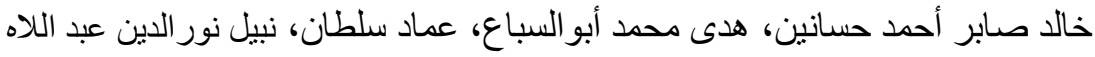

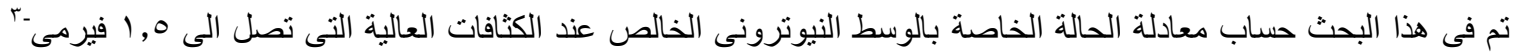

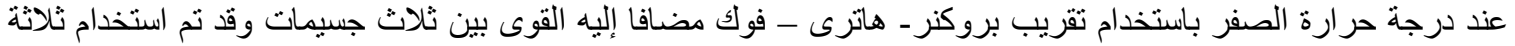

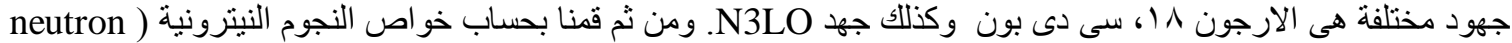
stars properties

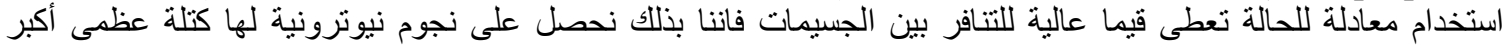

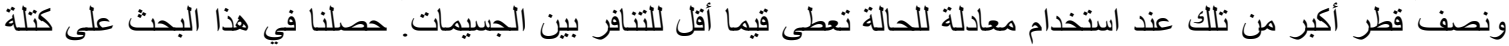
عظمى للنجوم النيوترونية تقترب من ضعف كتلة الثمس و هذا يتو افق مع القيم العملية المنشورة حديثا. 